\title{
Multi-objective optimal of surface-groundwater resources in the Dongxiezong irrigation district
}

\author{
Hengyue Yang ${ }^{1}$,Shaohui Zhang ${ }^{1}$,Wei Dai ${ }^{1}$,Yinong Li $^{1}$,Xin Zeng ${ }^{2}$ \\ ${ }^{1}$ State Key Laboratory of Simulation and Regulation of Water Cycle in River Basin, China Institute of Water Resources and \\ Hydropower Research, 20 West Chegongzhuang Rd., Beijing 100038, China. E-mail: 1183506777@qq.com; daiwei@iwhr.com \\ ${ }^{2}$ Water Recourses and Hydropower Planning and Design General Institute, Beijing 100120, China)
}

\begin{abstract}
: the water cycle in irrigation districts is extremely complicated under the dual influence of strong human activities and the nature. To establish the multi-water source rational allocation model of irrigation district, this paper first establish a multi-objective function based on economic utility, ecological utility and irrigation performance and improve Hicks optimization method. Then, combine it with chaotic particle swarm optimization algorithm to carry out research on temporal and spatial distribution evolution and optimal allocation of water resources in irrigation districts and collaborative scheduling and regulation of surface-groundwater. The multi-objective rational allocation is an important basis for the efficient use of water resources in irrigation districts and ecological harmony. This paper takes the typical irrigation area of Dongxiezong in Heilongjiang Province as the object for the study of the optimal allocation method of water resources in the irrigation district.
\end{abstract}

\section{Preface}

Single-objective optimization can offer one and only optimization result ${ }^{[1]}$. However, for multi-objective optimal allocation of water resources, it is difficult to obtain an optimal solution, and normally, a series of non-inferior solutions are obtained ${ }^{[2]}$. Sarker and Ray proposed a crowd-based approach for optimization. They solved two types of optimization problems using a multi-objective optimization model based on three different optimization methods ${ }^{[3]}$. Raju and Kumar established a multi-objective optimization model based on genetic algorithm in order to solve the problem of optimal reservoir scheduling while taking into consideration the crop planting structure ${ }^{[4]}$. Das and Datta developed a corresponding multi-objective management model group by establishing the relationship between a simulation model and an optimization model using embedding methods so as to use coastal aquifer groundwater more effectively ${ }^{[5]}$. Peralta and Datta developed a planning model by using embedded methods for regional sustainable income planning. The aim of this model is to maximize the total flow of the pump under the constraints that the pre-specified target water flow is stable and the damage on the existing planting structure is minimized ${ }^{[6]}$.

In recent years, Rezapour Tabari and Soltani established a multi-objective optimization model in order to maximize the reliability of the model system while minimizing the cost of water and aquifer backwater utilization. Compare the efficiency of the non-dominant sorting genetic algorithm (NSGA-II) with that of the sequence genetic algorithm (SGA), it can be seen that NSGA-II model can greatly reduce the computational cost of simulation optimization ${ }^{[7]}$. Bazargan-Lari et al. proposed a fuzzy multi-objective linear programming model based on the surface-groundwater joint configuration model featuring the conflict resolution method ${ }^{[8]}$. Rothmanand Mays established a multi-target GA model (MOGA) to assess the performance of sustainable water supply of the system. This model, taking into consideration the balance of the supply and demand of the total and underground, aiming at cost control, aquifer protection and growth, can make decisions on the water distribution of a certain area in a specific year ${ }^{[9]}$.

Artificial neural networks (ANN) are often used to build the tissue structure of the central nervous system ${ }^{[10]}$ ANN obtains an optimization plan through a learning process similar to that of the human brain. ANN is widely applied in many scientific fields for it creates a good approximation of a complex system through simple structures. Rao et al. established a conceptual regional joint planning model aiming to ensure sufficient water for crops so as to optimize the joint surface-groundwater allocation in the delta region of Eastern India. Rao et al. also proposed that the annealing algorithm can reduce the difficulty brought by the nonlinearity and non-convexity of the model itself. The combination of artificial neural network and high-efficiency algorithm of water flow

Corresponding author: 1183506777@qq.com 
movement stimulation can effectively reduce the amount of calculation caused by the water flow model. The results show that ANN solved by the annealing algorithm is effective in the study of model simulation of actual scale [11]. Safavi trained ANN to simulate the surface-groundwater interaction, and established a simulation optimization model by taking the genetic algorithm as the optimization model. In this way, the optimal configuration of surface-groundwater resources under multi-constraint conditions is achieved to meet as many requirements for irrigation as possible. This study shows that the simulation optimization model established by combining ANN with and genetic algorithm is very flexible, and can serve irrigation system management research under different constraints and assumptions ${ }^{[12]}$. Rao et al. has conducted similar researches and established simulation optimization simulation based on ANN for optimal management of the irrigation system.

When it comes to optimizing the usage of finite resources, the traditional optimization model based on Parato optimization theory is outperformed by the optimization model based on Hicks optimization theory in terms of global optimality. Therefore, this paper establishes a multi-water source optimal allocation model based on Hicks theory in order to improve the global optimality of the results.

\section{Multi-water source rational allocation model based on Hicks theory}

\subsection{Hicks Optimization Theory}

There is no dominant relationship between Hicks optimal solutions. Improving the performance of one target can cause decline of performance other target. During Hicks improvement, it is acceptable to improve the performance of targets by reducing the performance of a certain target as long as the overall performance of the system is improved. For practical problems in engineering, it is reasonable to reduce the performance of the subsystem to improve the overall performance when the decrease of performance is acceptable in order to obtain the optimal solution of the engineering system. Based on the definitions of Hicks dominance, Hicks optimal solution, Hicks optimal solution set, Hicks frontier, Hicks frontier, and Hicks utility loss obtained from related references and the multi-objective optimization problem described above, Hicks optimization method is improved (see Figure 1).

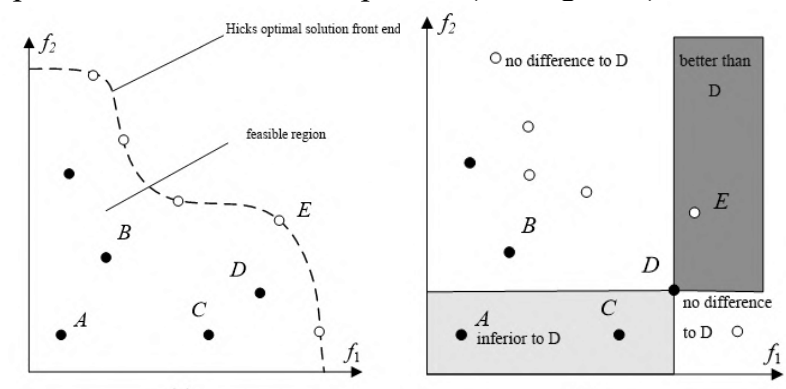

Figure 1, Principles of Hicks optimization.
Among all points (A, B, C, D, and E), E is the optimal one, and the decision variable e associated with it is not worse than any other decision variables. For e in this case, the performance of the indicator can no longer be improved, so solutions such as vector e can be regarded a Hicks optimal solution.

\begin{tabular}{|l|}
\hline The front end of Hicks optimal solutions \\
Feasible solutions \\
Same as D \\
Better than D \\
Worse than D \\
Same as D \\
\hline
\end{tabular}

\subsection{Model construction}

\subsubsection{Objective function}

The optimization objective function in this study is irrigation utility, ecological utility and irrigation performance, which is expressed as:

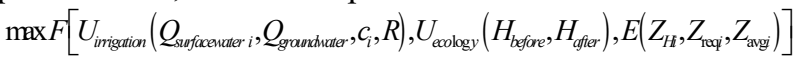

$$
\begin{aligned}
& U_{\text {surfacewater }}=\frac{p \eta_{\text {water }} Q_{\text {surfacewateri }} Q_{\text {surfacewater }}}{(\alpha+\lambda) Q_{\text {surfacewater }} \sum_{j=1}^{i} c_{j}+Q_{\text {surfacei }}\left(c_{0}+R\right)} \\
& U_{\text {groundwateri }}=\frac{p \eta_{\text {water }} Q_{\text {groundwater }}}{R_{i}} \\
& U_{\text {ecologyi }}=\frac{H_{\text {afteri }}}{H_{\text {beforei }}} \\
& E_{H i}= \begin{cases}\frac{Z_{\text {req } i}}{Z_{\text {avg } i}} \times 100 \% & Z_{H} \geq Z_{\text {req }} \\
\frac{Z_{H i}}{Z_{\text {avg } i}} \times 100 \% & Z_{H}<Z_{\text {req }}\end{cases}
\end{aligned}
$$

$Q_{\text {surface } i}$ water is the total amount of surface water $\left(\mathrm{m}^{3}\right)$ used in area $i ; Q_{\text {groundwater } i}$ is the total amount of ground water $\left(\mathrm{m}^{3}\right)$ used in area $i ; \alpha$ is the current value conversion factor, $\alpha=r(1+r)^{m} /\left[(1+r)^{m}-1\right]$. Since the operation and maintenance of the irrigation-drainage dual-use canal system is relatively simpler, the depreciation expense is used as the cost for the convenience of calculation. Assume dual-use canal irrigation systems have the same service life of $m$ years; $\lambda$ is the annual operating cost coefficient; $R$ is fuel cost, $R=\gamma$ Emnet, all $A T_{z} H / 102 \eta_{\text {pummp efficiency }} \eta_{\text {water }} T t$ with $\gamma$ being water soluble weight $\left(\mathrm{N} / \mathrm{m}^{3}\right) ; T_{z}$ is the actual service life of the pump (h); $H$ is the pump design head of delivery (m); $\eta$ is the pump installed efficiency; $E$ electricity price (yuan / $\mathrm{kWh}$ ); $T$ is the irrigation cycle (d); $t$ is the time for daily irrigation (h); Hgroundwater after irrigation $i$ is the groundwater level of point $i$ after irrigation (m); Hgroundwater before irrigation $i$ is the groundwater level of point $i$ before irrigation (m); Z Hi is the average irrigation depth of $1 / 2$ strip with the lowest average inflation water $(\mathrm{mm}) ; Z_{\text {reqi }}$ is the water required for irrigation at point $I(\mathrm{~mm}) ; Z_{\mathrm{avg}}$ is the average infiltration depth at point $i(\mathrm{~mm})$. 


\subsubsection{Constraints}

The constraints are:

$\sum_{i=1}^{I} \sum_{j=1}^{J} P A_{i, j} C N_{i, j} \leq Q_{\max }$

$\theta_{\min } \leq \theta_{0} \leq \theta_{f}$

$\theta_{\min } \leq \theta_{0}+\theta_{m} \leq \theta_{f}$

$\sum_{i=1}^{I} \sum_{j=1}^{J} P A_{i, j} \leq P A_{\max }$

$Q_{g}(u) \leq Q_{g \max } \quad \mathrm{u}=1,2, \ldots, \mathrm{R}$

$Q_{p}(u) \leq Q_{p \max } \quad \mathrm{u}=1,2, \ldots, \mathrm{R}^{\prime}$

$q_{g}(v) \leq q_{g \max } \quad \mathrm{u}=1,2, \ldots, \mathrm{r}$

$q_{p}(v) \leq q_{p \max } \mathrm{u}=1,2, \ldots, \mathrm{r}^{\prime}$

In formula (2), $Q_{\max }$ is the maximum amount of water in the irrigation process $\left(\mathrm{m}^{3}\right)$, and other parameters have the same meaning as above. $\theta \mathrm{min}$ is the lower limit of soil moisture content for crops $(\%), \theta_{0}$ is the initial water content $(\%), \theta_{\mathrm{m}}$ is the volumetric water content of soil increased under the quota of irrigation district (\%), and $\theta_{f}$ is the field water holding ratio (for dry land) or saturated water content (for paddy fields) $(\%) ; P A_{\max }$ is the total irrigation area $\left(\mathrm{mu}, 1 \mathrm{mu}=666.67 \mathrm{~m}^{3}\right) ; Q_{g}(\mathrm{u})$ is the actual water flow of the uth main canal in the research area during water delivery $\left(\mathrm{m}^{3} / \mathrm{s}\right), Q_{g \max }$ is the design water flow of the uth main canal $\left(\mathrm{m}^{3} / \mathrm{s}\right), R$ is the number of main canals in the research area (unit: strip); $Q_{p}(\mathrm{u})$ is the actual water flow of the uth main canal in the research area during drainage $\left(\mathrm{m}^{3} / \mathrm{s}\right), Q_{p \max }$ is the increased water flow of the uth main canal $\left(\mathrm{m}^{3} / \mathrm{s}\right), R^{\prime}$ is the number of main dual-purpose canals for irrigation and drainage in the research area (strip); $q_{g}(\mathrm{v})$ is the actual flow of the $v$ th branch channel in the research area rate during water delivery $\left(\mathrm{m}^{3} / \mathrm{s}\right), q_{\text {gmax }}$ is the design flow of the $v$ th branch channel $(\mathrm{m} 3 / \mathrm{s}), r$ is the number of branch canals in the research area (strip); $q_{p}(\mathrm{v})$ is the actual flow of the $v$ th branch channel in the research area rate during drainage $\left(\mathrm{m}^{3} / \mathrm{s}\right), q_{p \max }$ is the increased flow of the $v$ th branch channel $\left(\mathrm{m}^{3} / \mathrm{s}\right), r^{\prime}$ is the number of branch dual-purpose canals for irrigation and drainage in the research area (unit: strip).

There are two objectives for optimization, namely, maximum effectiveness of the irrigation system and optimal irrigation performance. The objective function to be optimized, together with the many constraints, constitutes a multi-objective optimization model for allocating irrigation water in the research area. After that, the model needs to be instantiated according to the actual situation of the research area and the space and attribute data collected and processed in the early stage. The optimized model is solved with the appropriate multi-objective intelligent algorithm so as to obtain the optimal irrigation plan in the research area.

\section{Model solution}

\subsection{Fast non-dominated sorting}

Different weights are assigned to the three sub-goals in the objective function according to different requirements, and the integrated objective function of each water demand node can be expressed as:

$$
F_{M i}=\alpha_{1} U_{\text {irrigate }}-\alpha_{2} U_{\text {ecolog } y}-\alpha_{3} E
$$

In this formula: $\alpha 1, \alpha 2$, and $\alpha 33$ are respectively the weights of the irrigation utility function, the ecological utility function, and the irrigation performance function $(\alpha 1+\alpha 2+\alpha 3=1)$. When the three are considered to be equally important, $\alpha_{1}=\alpha_{2}=\alpha_{3}$; when the utility is considered to be the most important, the risk the second, and the loss the least, $\alpha_{1}>\alpha_{2}>\alpha_{3}$, and so on.

The overall integrated objective function of the pipe network system is expresses as:

$$
F_{M}=\sum_{i=1}^{n} F_{M i}
$$

Each offspring $Q_{t}$ and its parent $P_{t}$ are sorted according to the total objective function value of the system. The larger the function value, the better. The division of the non-dominated set is similar to that of the traditional NSGA-II.

\subsection{Congestion calculation}

(1) The congestion degree id of each point is set to 0 ;

(2) Apply non-dominated sorting to each target, set the congestion of the two individuals on the boundaries as infinite, that is $O_{d}=I_{d}=\infty$;

(3) The degree of freedom of the remaining individuals is calculated according to formula (5)

$$
i_{d}=\sum_{j=1}^{3}\left(\left|f_{j}^{i+1}-f_{j}^{i-1}\right|\right)
$$

$i_{d}$ is the congestion degree of point $i ; f_{j}^{i+1}$ is the $j$ th objective function value of point $i+1$, and $f_{j}^{i-1}$ is the $j$ th objective function value of point $i-1$.

\subsection{Congestion comparison operator}

After applying fast non-dominated sorting and congestion calculation, each individual in the group has two attributes: the non-dominated order $i_{\text {rank }}$ determined by the non-dominated sorting and the congestion degree $i_{d}$. The congestion comparison operator based on the above two attributes should meet the following two conditions:

(1) If the non-dominated layer of individual $i$ is superior to the non-dominated layer of individual $j$, that is $i_{\text {rank }}<j_{\text {rank }}$;

(2) If they are in the same level, and individual $i$ has a larger congestion distance than individual $j$, that is $i_{\text {rank }}=j_{\text {rank }}$ and $i_{d}>j_{d}$.

When both of the above conditions are met, individual $i$ is superior to individual $j$.

\subsection{Algorithm procedure}


Initial contemporary population $\mathrm{Gen}=0$, which generates initial population $P_{0}$ and produce offspring $Q_{0}$ according to crossover and mutation; the number of the individuals is $N$.

The following operations are performed when the evolutionary algebra $\mathrm{Gen}<\mathrm{Gen}_{\max }$.

(1) Fast non-dominated sorting. Put the offspring $Q_{t}$ and its parent $P_{t}$ into the set $R_{t}$ (the number of individuals is $2 \mathrm{~N}$ ). Perform non-dominated sorting to the elements in $R_{t}$ according to the evaluation standard of total integrated objective function value of the pipe network system expressed in formula (4-34), thereby form a non-dominated sequence $Z_{i}$.

(2) Form an elite population. Put it into the elite population according to the number of layer of $Z_{i}$ and the two conditions in congestion comparison operator until the number of individuals in the elite population reaches the upper limit $N$. At this moment, the elite population is recorded as a new parent $P_{t}+1$.

(3) Crossover and mutation. A new offspring $Q_{t+1}$ is generated based on crossover and mutation.

(4) $\mathrm{Gen}=\mathrm{Gen}+1$, enter the next cycle

\section{Model application}

\subsection{Overview of the research area}

The main irrigation area of Dongxiezong lies in Youyi County, Heilongjiang Province. It is located in the southeast of Jiamusi City, in the northeast of Shuangyashan City, and on the edge of a large swamp in the Sanjiang Plain. In the typical area, there are a total of 2 branch canals, with a total length of $14.498 \mathrm{~km}$; 18 canals with a total length of $48.735 \mathrm{~km}$ (including 7.305 $\mathrm{km}$ for 2 single irrigation channels and $41.430 \mathrm{~km}$ for 16 irrigation-drainage channels); 174 agricultural canals with a total length of $108.094 \mathrm{~km}$ (including $66.769 \mathrm{~km}$ for 122 single irrigation canals and $41.324 \mathrm{~km}$ for 52 irrigation-drainage canals); the branch travels from north to south with the main tributary ditches, head ditches and field ditches lying vertical with each other. The distance between two nearest head ditches is about $1000 \mathrm{~m}$, while for field ditches, it is about $200 \mathrm{~m}$. See Figure 2.

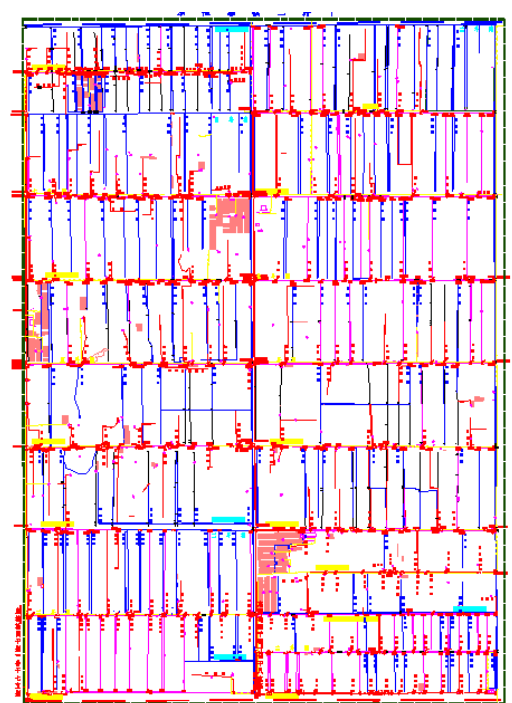

Figure 2,Canal layout in the research area canal layout.

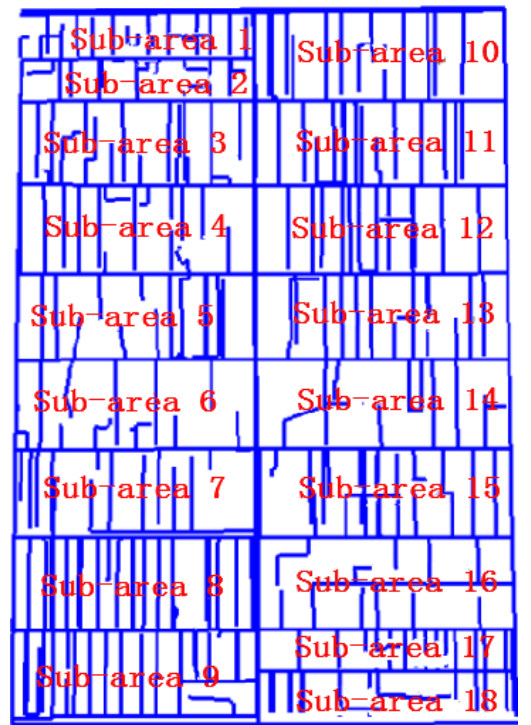

Figure 3,Sub-areas in typical irrigation area

Divide the typical area into 18 sub-areas according to the controlled area of the head ditch, as is shown in Figure 3.

(2) Parameter design of channels in the typical zone

The area of controlled drainage is $67,800 \mathrm{mu}$. The ditches correspond to the channels in the typical area. Set 2 tributary ditches with a length of $16.326 \mathrm{~km}$ and 7 branch ditches with a total length of $15.076 \mathrm{~km}$. Set 127 agricultural field ditches under head ditches, with a total length of about $100.488 \mathrm{~km}$.

(3) Plant the crop

The crop selected in the typical areas is rice.

(4) Water source project

The main irrigation area of Dongxie is a newly-built one with 6 canal heads (including 3 intake gates and 3 pumping stations). According to irrigation water sources, divided it into three sub-areas, namely, general main canal area of the Yinsong, main canal area of Sanhuanpao, and main canal area of Naoli River. In this research, the typical area is in general main canal area of the Yinsong with one water project and $40 \mathrm{~m}^{3} / \mathrm{s}$ of water intake. 


\subsection{Optimization results}

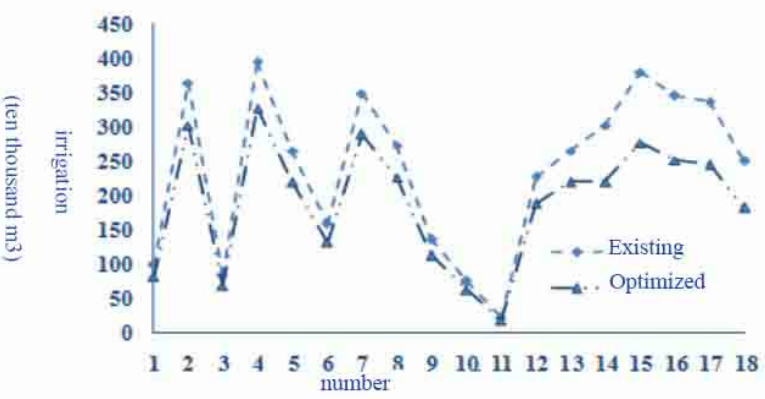

(a)

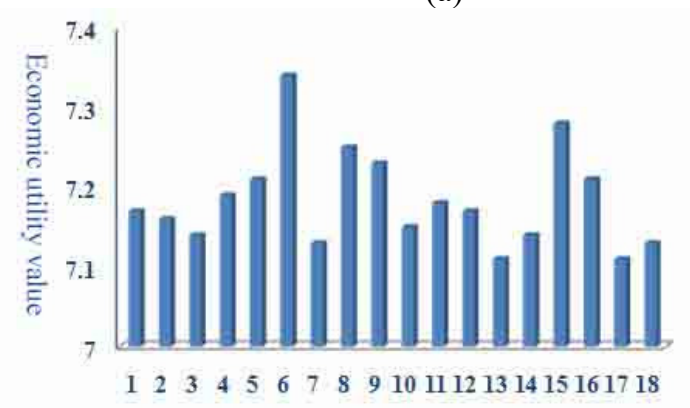

(b)

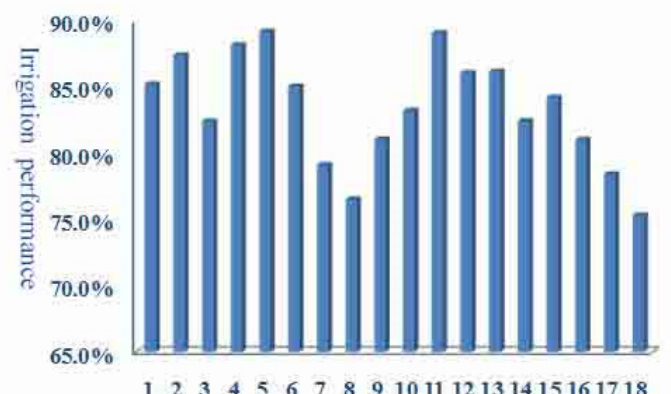

(c)

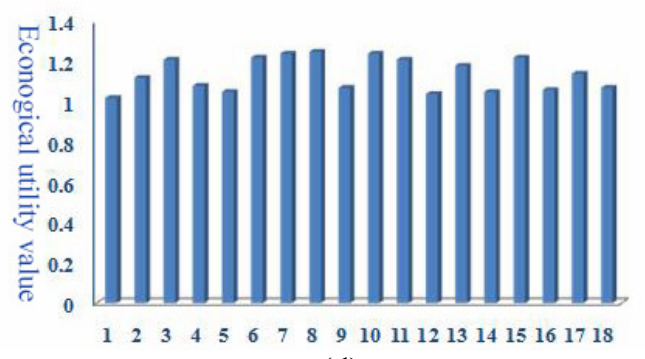

(d)

Figure 4, Result of irrigation area optimization

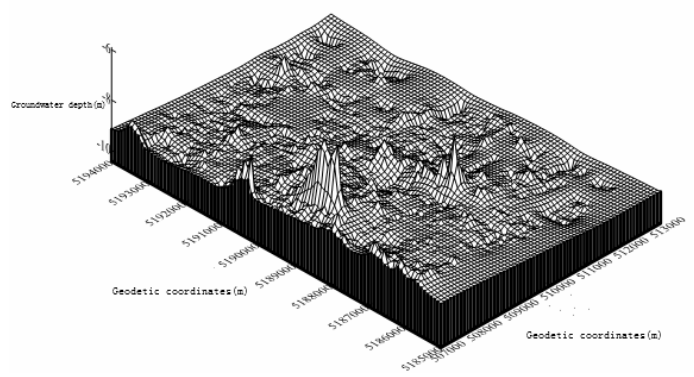

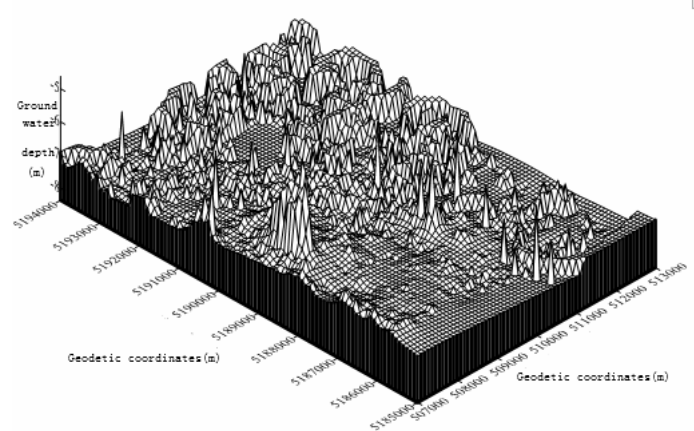

Figure 5,Comparison of groundwater level before and after optimization

\section{Conclusion}

Three objectives are considered simultaneously during the optimization of water resources in the irrigation area in this study, so it is a multi-objective optimization problem in the field of water resources optimization and scheduling. Therefore, this paper first expounds some concepts and basic theories about multi-objective optimization and displays mathematical expressions accordingly, including the composition of objective functions, decision variable space, constraints, etc. Based on the exploration of multi-objective optimization, this paper defines optimal Hicks and solutions to multi-objective optimization, which is part of the definition of Hicks optimal solution. A multi-objective optimization model with economic utility, ecological utility and irrigation performance as its target is established and applied in the typical area of the main irrigation area of Dongxie. Compared with the existing optimal allocation model of irrigation water resources, the multi-objective optimization model helps to save water by $17 \% \sim 27 \%$ and pull up groundwater level by $2 \%$ to $22 \%$.

\section{Acknowledgments}

This research is supported by National key R\&D Program of China(2017YFC0403201and 2017YFC0403204)

\section{Reference}

1. Cohon J L, Marks D H. A review and evaluation of multiobjective programing techniques. Water Resources Research, 1975, 11(11):208-220.

2. Park C H, Aral M M. Multi-objective optimization of pumping rates and well placement in coastal aquifers. Journal of Hydrology, 2004, 290(1-2):80-99.

3. Sarker Ruhul, Ray Tapabrata. An improved evolutionary algorithm for solving multi-objective crop planning models. Computers and Electronics in Agriculture, 2009, 68(2):191-199.

4. 4.K.Srinivasa Raju, D. Nagesh Kumar. Irrigation Planning using Genetic Algorithms. Water 
Resources Management, 2004, 18(2):163-176.

5. Das A, Datta B. Development of Management Models for Sustainable Use of Coastal Aquifers. Journal of Irrigation \& Drainage Engineering, 1999, 125(3):112-121.

6. Richard C. Peralta, Bithin Datta. Reconnaissance-Level Alternative Optimal Ground-Water Use Strategies. Journal of Water Resources Planning and Management, 1990, 116(5):676-692.

7. Tabari M M R, Soltani J. Multi-Objective Optimal Model for Conjunctive Use Management Using SGAs and NSGA-II Models. Water Resources Management, 2013, 27(1):37-53.

8. Bazarganlari M R, Kerachian R, Mansoori A. A conflict-resolution model for the conjunctive use of surface

9. Mays L W. Groundwater Resources Sustainability:
Past, Present, and Future. Water Resources Management, 2013, 27(13):4409-4424.

10. Rogers L L, Dowla F U. Optimization of Ground Water Remediation Using Artificial Neural Networks. Water Resources Research, 1994, 30(2):457-481.

11. Rao S V N, Bhallamudi S M, Thandaveswara B S, et al. Conjunctive Use of Surface and Groundwater for Coastal and Deltaic Systems. Journal of Water Resources Planning \& Management, 2004, 130(3):255-267.

12. Safavi $H \quad R$, Darzi $F$, Mariño $M \quad A$. Simulation-Optimization Modeling of Conjunctive Use of Surface Water and Groundwater. Water Resources Management, 2010, 24(10):1965-1988.

13. Rao $\mathrm{S}$ V N. Optimal Pumping from Skimming Wells. Journal of Hydrologic Engineering, 2006, 11(5):464-471. 